\title{
NUEVA ESPECIE DE VESPERUS DEJEAN, 1821 DE LA PENÍNSULA IBÉRICA (COLEOPTERA, CERAMBYCIDAE)
}

\author{
P. Bercedo Páramo (*) y P. Bahillo de la Puebla (**)
}

\begin{abstract}
RESUMEN
Se describe Vesperus jertensis sp. nov. del valle del Jerte (provincia de Cáceres, España) y se compara con las especies afines del género. De $V$. serranoi, la especie más próxima, se separa por la mayor longitud antenal y la superficie cefálica, pronotal y elitral que es brillante en la nueva especie debido a una menor densidad de su puntuación. Del resto de las especies de Vesperus se separa, entre otros caracteres, porque la distancia interocular es mayor que la distancia entre las inserciones antenales. Además se definen dos grupos de especies, el grupo de $V$. brevicollis y el grupo de $V$. conicicollis, atendiendo a caracteres de la morfología cefálica y de la armadura genital masculina.

Palabras clave: Coleoptera, Cerambycidae, taxonomía, Vesperus jertensis sp. nov., grupos de especies, España.
\end{abstract}

\section{ABSTRACT \\ New species of Vesperus Dejean, 1821 from the Iberian Peninsula (Coleoptera, Cerambycidae)}

Vesperus jertensis sp. nov. from the Jerte river valley (Cáceres prov., Spain) is described and compared with the closest allies in the genus. It differs from the closest species, $V$. serranoi, by the longer antennae and the brighter head, pronotum and elytral surface because of the lesser density of punctures. From the remainder of the species of Vesperus, it differs, among other characters, by the interocular distance being wider than the interantennal one. Moreover, two groups of species are defined based upon characters of head morphology and male genitalia, the $V$. brevicollis group and the $V$. conicicollis group.

Key words: Coleoptera, Cerambycidae, taxonomy, Vesperus jertensis sp. nov., species groups, Spain.

\section{Introducción}

El género Vesperus Dejean, 1821 se halla integrado por especies muy polimorfas y con marcado dimorfismo sexual con machos alados y hembras braquípteras, normalmente de mayor tamaño que los machos y con el abdomen hipertrofiado. Después de la importante revisión del género reali- zada por Villiers (1974), se han descrito en la Península Ibérica: Vesperus serranoi Zuzarte, 1985, Vesperus baesuriensis Zuzarte, 1985 (Zuzarte, 1985; Bense, 1995) y Vesperus joanivivesi Vives, 1998 (Vives, 1998), lo que eleva a 11 el número de especies de este género en nuestra Península.

En un lote de coleópteros capturados en la provincia de Cáceres en 1997 por D. Ángel Blázquez y

* C/ Ferrer, 23, Vegas del Condado, 24153, León, España.

** C/ Ibaizabal, 1, $1^{\circ}$ C; 48901 Barakaldo, Vizcaya, España. 


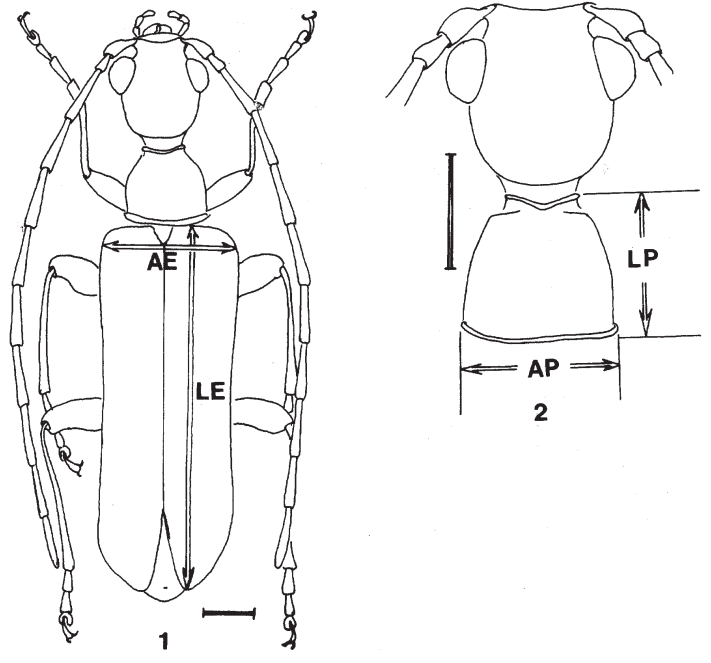

Figs. 1-2.- Vesperus jertensis sp. nov., macho. 1) silueta y esquema de medidas elitrales; 2) esquema de las mediciones pronotales. Escala $=1 \mathrm{~mm}$.

Figs. 1-2.- Vesperus jertensis sp. nov., male: 1) outline and elytral measurements; 2) pronotal measurements, schematic. Scale $=1 \mathrm{~mm}$.

comunicados al primero de los autores para su estudio, se detectaron tres ejemplares de un pequeño Vesperus de Plasencia, con unos caracteres que no se ajustaban a las especies conocidas hasta la fecha. Los mencionados ejemplares fueron determinados por D. Eduard Vives como Vesperus sanzi Reitter, 1895. Con posterioridad se recibieron otros 11 ejemplares con las mismas características, procedentes de la misma localidad, contenidos en la colección particular de D. Jesús de Castro y determinados como Vesperus conicicollis Fairmaire et Coquerel, 1866. Durante una excursión entomológica realizada en agosto de 1998 por tierras extremeñas se capturaron 130 ejemplares más del mismo Vesperus. El estudio de estos 144 ejemplares, nos ha llevado a confirmar la constancia de una serie de caracteres distintivos con respecto a las demás especies del género que nos permiten considerar los ejemplares de Plasencia como pertenecientes a un nuevo taxon que describimos a continuación.

\section{Vesperus jertensis sp. nov.}

Material tíPICO: $144 \sigma^{7} \sigma^{7}$ Plasencia, Cáceres (España), cuadrícula U.T.M.: 29TQE43.

Holotipo: $1 \sigma^{7}$ provisto de las siguientes etiquetas: Etiqueta blanca mecanografiada: Plasencia (CC), 11-VIII-1998,

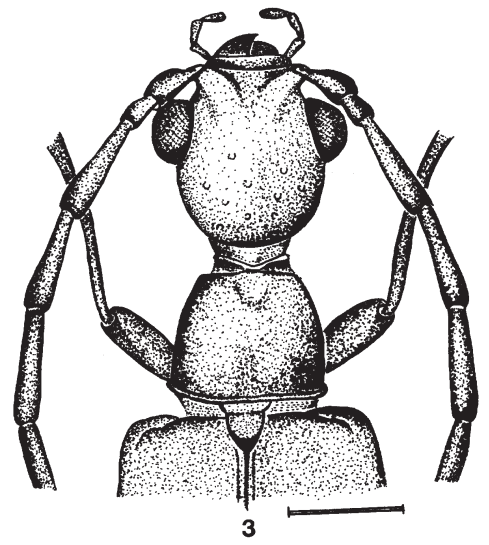

Fig. 3.- Vesperus jertensis sp. nov., macho: cabeza y pronoto, visión dorsal. Escala $=1 \mathrm{~mm}$.

Fig. 3.- Vesperus jertensis sp. nov., male: head and pronotum, dorsal view. Scale $=1 \mathrm{~mm}$.

29TQE43, P. Bercedo Leg. / Etiqueta anaranjada mecanografiada: HOLOTYPUS, Vesperus jertensis Bercedo \& Bahillo, Bercedo \& Bahillo des. 1998; depositado en el Museo Nacional de Ciencias Naturales de Madrid (MNCN).

PARATIPOS: $143 \sigma^{\pi} \sigma^{\pi}$ de la misma localidad. 11 con etiqueta blanca manuscrita: Plasencia (luz), 1-IX-1996 / etiqueta blanca manuscrita: Cáceres, J. de Castro leg. / Etiqueta blanca manuscrita: Vesperus conicicollis; 3 con etiqueta blanca manuscrita: Plasencia, Cáceres 29-VIII-1997, A. Blázquez leg. / Etiqueta blanca manuscrita: Vesperus sanzi Rtt. E. Vives det. 1997; 129 con etiqueta blanca mecanografiada: Plasencia (CC), 11-VIII-1998, 29TQE43, P. Bercedo Leg.

A todos los paratipos se les adjunta una etiqueta anaranjada mecanografiada con las siguientes indicaciones: PARATYPUS, Vesperus jertensis Bercedo \& Bahillo, Bercedo \& Bahillo des. 1998.

El total de paratipos quedan depositados en las siguientes colecciones: MNCN (Madrid) 20 ex.; J. de Castro (PlasenciaCáceres) 11 ex.; A. Blázquez (Plasencia-Cáceres) 4 ex.; A. J. Zuzarte (Monforte-Portugal) 6 ex.; P. Bahillo (BarakaldoVizcaya) 34 ex. y el resto en la colección P. Bercedo-L. Arnáiz (Vegas del Condado-León).

Diagnosis: Vesperus de pequeño tamaño $(7,5-11,8 \mathrm{~mm})$, con tegumentos brillantes de coloración variable de amarillo-pajiza a pardo oscura casi negra. Antenas que sobrepasan ligeramente el ápice elitral. Espacio interocular mayor que la separación de las inserciones antenales.

DESCRIPCIÓN DEL MACHO (Fig. 1): 7,5-11,8 mm (incluida la cabeza). Coloración general de los tegumentos variable, de amarillo-pajiza a marrón oscura, casi negra en algunos ejemplares. 

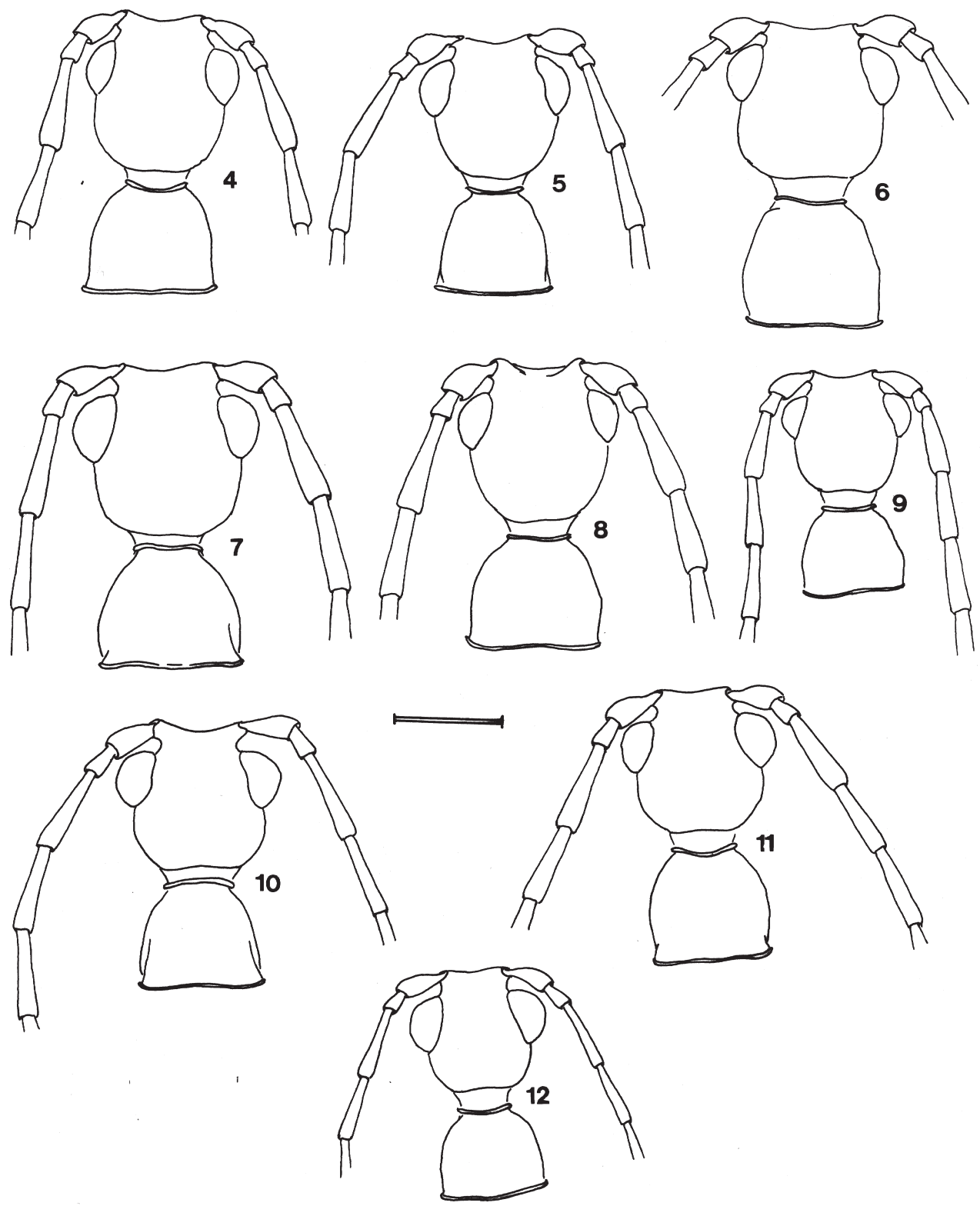

Figs. 4-12.- Vesperus jertensis sp. nov., macho: variabilidad de la morfología cefálica, 4) HOLOTYPUS. Escala= $1 \mathrm{~mm}$.

Figs. 4-12.- Vesperus jertensis sp. nov., male: variability of head morphology, 4) HOLOTYPUS. Scale=1 mm.

Cabeza (Fig. 3) con superficie lisa y brillante, con algunos puntos gruesos dispersos y muy escasos. En la superficie cefálica se observan sedas en número reducido algo más abundantes en las áreas postoculares y en la frente. Espacio interantenal cóncavo, sin surco medio definido, siendo la separación de las inserciones antenales más corta que la separación interocular. Antenas de 11 artejos que sobrepasan ligeramente el ápice elitral. Los ojos son pequeños, sin escotadura, glabros, moderadamente salientes y más o menos hemisféricos. 
Pronoto variable, desde ligeramente transverso en algunos ejemplares hasta ligeramente más largo que ancho en otros ( $\mathrm{LP} / \mathrm{AP}=0,82-1,14)$; rebordeado anterior y posteriormente. El borde anterior apreciablemente escotado. Superficie pronotal como la cefálica, brillante y con algunos puntos gruesos dispersos muy escasos de los que parten sendas sedas cortas. Escudete con el ápice regularmente redondeado y rebordeado en toda su periferia siendo especialmente patente el reborde en los laterales del mismo. Superficie del escudete lisa y brillante con algunas sedas dispersas. Élitros de lados paralelos, aproximadamente 2,5 veces más largos que anchos considerados en conjunto y a la altura de la región humeral $(\mathrm{LE} / \mathrm{AE}=$ 2,08-2,82). Superficie elitral con algunos pelos dispersos tumbados hacia atrás. La pilosidad, que es muy escasa, se hace ligeramente más densa en el tercio apical de los élitros. Sutura elitral finamente rebordeada. Patas y abdomen como en el resto de los Vesperus.

Pigidio semicircular con la región apical del mismo ligeramente truncada (Fig. 16). Tegmen (Figs. 13, 14 y 15) proporcionalmente corto, con lóbulos parameroides alargados y relativamente largos. Pene con el ápice del tectum (placa dorsal) truncado y escotado (Fig. 17).

\section{Hembra: Desconocida.}

ETimología: El nombre de la especie hace referencia al río Jerte en cuyas riberas, en la localidad de Plasencia (Cáceres, España), se han capturado la totalidad de los ejemplares que componen la serie típica.

VARIABILIDAD: Los ejemplares examinados se manifiestan variables como el resto de los representantes del género Vesperus. Esta variabilidad se puede observar en todas la características morfológicas.

En lo referente a la coloración de los tegumentos, encontramos desde ejemplares amarillo pajizos, hasta ejemplares con los tegumentos de color marrón muy oscuro casi negro, pasando por ejemplares con todas las coloraciones intermedias entre ambos extremos.

Para cuantificar la variabilidad en las dimensiones corporales del nuevo Vesperus se ha realizado un estudio biométrico sobre una muestra, representativa del total de los integrantes de la serie típica, formada por 45 ejemplares entre los cuales se incluyen los ejemplares de mayor y menor tamaño.
En todos los ejemplares estudiados se tomaron las siguientes medidas tal y como se refleja en las figuras 1 y 2 :
$\mathrm{AP}=$ Anchura máxima del pronoto.
$\mathrm{LP}=$ Longitud máxima del pronoto.
$\mathrm{AE}=$ Máxima anchura elitral conjunta.
$\mathrm{LE}=$ Longitud máxima de los élitros.

Los resultados obtenidos en estas mediciones (Tabla 1) reflejan una discreta variabilidad en el tamaño de los ejemplares. Especialmente llamativo, resulta el hecho de que la relación LP/AP que habitualmente se viene utilizando en las claves de determinación de los representantes del género Vesperus para separar grupos de especies, es notablemente variable, al menos en el taxon que nos ocupa, fluctuando alrededor de valores próximos a 1 pero encontrando valores que oscilan entre 0,82 (mínimo detectado) y 1,14 (máximo detectado).

En el mismo sentido la relación entre la longitud y la anchura elitral LE/AE, se manifiesta asimismo variable, fluctuando alrededor de un valor de 2,49 pero oscilando entre un mínimo de 2,08 y un máximo de 2,82 .

Tal y como se refleja en las figuras 4-12, la forma de la cabeza es también muy variable encontrándose ejemplares con los lados de la cabeza divergentes, paralelos o con la región cefálica posterior redondeada. No obstante, en todos los ejemplares examinados, a pesar de la gran variabilidad cefálica, la separación entre los ojos es siempre mayor que la separación entre las inserciones antenales.

La longitud de la antenas (Fig. 1) es ligeramente variable de unos ejemplares a otros pero siempre sobrepasando el ápice elitral. La longitud relativa del tercer, cuarto y quinto antenómero, que fué utilizado por Zuzarte (1985) como elemento diagnóstico en la descripción de Vesperus serranoi Zuzarte, 1985, es también un carácter variable. De manera general en $V$. jertensis el tercer antenómero es más largo que el cuarto y que el quinto, pero también hemos estudiado ejemplares en los que esto no se cumple (Fig. 9).

La armadura genital masculina, en lo que se refiere a la forma del tegmen y lóbulos parameroides (Figs. 13-15) se manifiesta asimismo muy variable. Lo mismo se puede afirmar de la pilosidad de los lóbulos parameroides aunque presente un patrón único de distribución caracterizado por la presencia de macroquetas (en número variable) en la región apical de los lóbulos parameroides y una 
Tabla 1.- Medidas de 45 ejemplares de Vesperus jertensis sp. nov., tomadas como se ve en las figuras 1 y 2 . Relaciones morfométricas: $\mathrm{LP} / \mathrm{AP}=$ Relación longitud/anchura pronotal. $\mathrm{LE} / \mathrm{AE}=$ Relación longitud/anchura elitral. $\mathbf{M}, \mathbf{m}, \mathbf{x}$ y $\boldsymbol{\sigma}=$ Valores máximo, mínimo, media y desviación típica de cada parámetro.

Table 1.- Measurements of 45 specimens of Vesperus jertensis sp nov., taken as in figures 1 and 2. Morphometric ratios. $\mathrm{LP} / \mathrm{AP}=$ Pronotal length/width ratio. $\mathrm{LE} / \mathrm{AE}=$ Elitral length/width ratio. $\mathbf{M}, \mathbf{m}, \mathbf{x}$ y $\boldsymbol{\sigma}=$ Maximum, minimum, mean and standard deviation of each parameter.

\begin{tabular}{lllllll}
\hline $\mathbf{N}^{\mathbf{~}}$ & LP & AP & LE & AE & LP/AP & LE/AE \\
\hline $\mathbf{1}$ & 1,15 & 1,25 & 5,4 & 2,1 & 0,92 & 2,57 \\
$\mathbf{2}$ & 1,5 & 1,6 & 7,1 & 3 & 0,94 & 2,37 \\
$\mathbf{3}$ & 1,55 & 1,6 & 6,7 & 2,8 & 0,97 & 2,39 \\
$\mathbf{4}$ & 1,55 & 1,7 & 7,1 & 2,9 & 0,91 & 2,45 \\
$\mathbf{5}$ & 1,6 & 1,65 & 7 & 2,8 & 0,96 & 2,5 \\
$\mathbf{6}$ & 1,4 & 1,6 & 6,9 & 2,85 & 0,87 & 2,42 \\
$\mathbf{7}$ & 1,4 & 1,5 & 6,5 & 2,6 & 0,93 & 2,5 \\
$\mathbf{8}$ & 1 & 1,2 & 5 & 2 & 0,83 & 2,5 \\
$\mathbf{9}$ & 1,4 & 1,4 & 6,9 & 2,45 & 1 & 2,82 \\
$\mathbf{1 0}$ & 1,65 & 1,7 & 7,3 & 2,8 & 0,97 & 2,61 \\
$\mathbf{1 1}$ & 1,75 & 1,8 & 7,8 & 3,05 & 0,97 & 2,56 \\
$\mathbf{1 2}$ & 1,8 & 1,65 & 7,9 & 3,2 & 1,09 & 2,47 \\
$\mathbf{1 3}$ & 1,15 & 1,25 & 5,8 & 2,2 & 0,92 & 2,64 \\
$\mathbf{1 4}$ & 1,75 & 1,75 & 7,3 & 2,95 & 1 & 2,47 \\
$\mathbf{1 5}$ & 1,6 & 1,6 & 6,5 & 2,7 & 1 & 2,41 \\
$\mathbf{1 6}$ & 1,55 & 1,55 & 6,75 & 2,7 & 1 & 2,5 \\
$\mathbf{1 7}$ & 1,55 & 1,55 & 6,7 & 2,7 & 1 & 2,48 \\
$\mathbf{1 8}$ & 1,75 & 1,65 & 7 & 2,8 & 1,06 & 2,5 \\
$\mathbf{1 9}$ & 1,15 & 1,15 & 5,2 & 2,05 & 1 & 2,54 \\
$\mathbf{2 0}$ & 1,2 & 1,25 & 5,5 & 2 & 0,96 & 2,75 \\
$\mathbf{2 1}$ & 1,6 & 1,65 & 6,85 & 2,8 & 0,97 & 2,45 \\
$\mathbf{2 2}$ & 1,5 & 1,5 & 6,6 & 2,7 & 1 & 2,44 \\
$\mathbf{2 3}$ & 1,3 & 1,4 & 6 & 2,3 & 0,93 & 2,61 \\
$\mathbf{2 4}$ & 1,4 & 1,4 & 6 & 2,35 & 1 & 2,55 \\
$\mathbf{2 5}$ & 1,35 & 1,4 & 5,7 & 2,45 & 0,96 & 2,33 \\
$\mathbf{2 6}$ & 1,4 & 1,55 & 6,55 & 2,7 & 0,90 & 2,43 \\
$\mathbf{2 7}$ & 1,15 & 1,3 & 5,55 & 2,15 & 0,88 & 2,58 \\
$\mathbf{2 8}$ & 1,2 & 1,25 & 5,5 & 2,2 & 0,96 & 2,5 \\
$\mathbf{2 9}$ & 1,45 & 1,75 & 7,2 & 2,95 & 0,82 & 2,44 \\
$\mathbf{3 0}$ & 1,45 & 1,5 & 6,45 & 2,6 & 0,97 & 2,48 \\
$\mathbf{3 1}$ & 1,65 & 1,7 & 7,15 & 2,85 & 0,97 & 2,50 \\
$\mathbf{3 2}$ & 1,4 & 1,5 & 6,3 & 2,5 & 0,93 & 2,52 \\
$\mathbf{3 3}$ & 1,7 & 1,7 & 7,35 & 3,1 & 1 & 2,37 \\
$\mathbf{3 4}$ & 1,6 & 1,8 & 7,4 & 3 & 0,89 & 2,47 \\
$\mathbf{3 5}$ & 1,3 & 1,35 & 6,95 & 2,65 & 0,96 & 2,62 \\
$\mathbf{3 6}$ & 1,5 & 1,6 & 7,5 & 2,9 & 0,95 & 2,59 \\
$\mathbf{3 7}$ & 1,25 & 1,4 & 6,65 & 2,4 & 0,89 & 2,77 \\
$\mathbf{3 8}$ & 1,35 & 1,6 & 6,6 & 2,85 & 0,84 & 2,32 \\
$\mathbf{3 9}$ & 1,3 & 1,55 & 6,65 & 2,45 & 0,84 & 2,71 \\
$\mathbf{4 0}$ & 1,5 & 1,65 & 7,3 & 2,95 & 0,90 & 2,47 \\
$\mathbf{4 1}$ & 1,5 & 1,6 & 7,25 & 3 & 0,94 & 2,42 \\
$\mathbf{4 2}$ & 1,3 & 1,55 & 7,1 & 2,75 & 0,84 & 2,58 \\
$\mathbf{4 3}$ & 1,7 & 1,5 & 7,3 & 3,05 & 1,14 & 2,39 \\
$\mathbf{4 4}$ & 1,6 & 1,7 & 7,05 & 2,95 & 0,94 & 2,39 \\
$\mathbf{4 5}$ & 1,25 & 1,5 & 6,15 & 2,95 & 0,83 & 2,08 \\
\hline $\mathbf{M}$ & 1,8 & 1,8 & 7,9 & 3,2 & 1,14 & 2,82 \\
$\mathbf{m}$ & 1 & 1,15 & 5 & 2 & 0,82 & 2,08 \\
$\mathbf{X}$ & 1,45 & 1,52 & 6,65 & 2,67 & 0,94 & 2,49 \\
$\mathbf{\sigma}$ & 0,19 & 0,17 & 0,69 & 0,32 & 0,07 & 0,13 \\
\hline & & & & & &
\end{tabular}

serie de microquetas de abundancia y distribución variable en la superficie de estos.

\section{Discusión}

El nuevo Vesperus es fácilmente separable de todos los demás Vesperus ibéricos.

De Vesperus aragonicus Baraud, 1964 y Vesperus bolivari Oliveira, 1853, con tamaños que sobrepasan los $20 \mathrm{~mm}$, el nuevo Vesperus se separa "de visu" por su tamaño considerablemente menor (con un tamaño máximo de $11,8 \mathrm{~mm}$ ). Por otro lado la separación entre las inserciones antenales de los primeros es mayor que la distancia interocular mientras que en $V$. jertensis es menor.

De Vesperus xatarti Dufour, 1839 y Vesperus fuentei Pic, 1905 se separa por presentar ambos el espacio interantenal surcado frente a un espacio regularmente cóncavo en la nueva especie. Además en $V$. xatarti y $V$. fuentei el espacio interocular es menor que la separación de las inserciones antenales mientras que en $V$. jertensis es mayor.

En el resto de las especies ibéricas del género Vesperus, se pueden definir dos grupos atendiendo a la morfología cefálica y a la conformación de su armadura genital:

1.- "El grupo de V. brevicollis" integrado por Vesperus brevicollis Graëlls, 1858, Vesperus sanzi Reitter, 1895 y Vesperus joanivivesi Vives, 1998, que se caracteriza por presentar el espacio interantenal surcado, ápice de la placa dorsal (tectum) del pene redondeado (Figs. $20 \mathrm{PN}$ y 22) y tegmen largo con lóbulos parameroides proporcionalmente cortos y rechonchos (Fig. 20).

2.- "El grupo de $V$. conicicollis" integrado por Vesperus conicicollis Fairmaire et Coquerel, 1866, Vesperus baesuriensis Zuzarte, 1985, Vesperus serranoi Zuzarte, 1985 y Vesperus jertensis sp. nov., que se caracteriza por presentar el espacio interantenal cóncavo, no surcado, el ápice del tectum truncado y más o menos cóncavo o escotado (Figs. 17, 18PN, $19 \mathrm{PN}$ y 21) y un tegmen proporcionalmente más corto con lóbulos parameroides más alargados (Figs. 13, 14, 15, 18, y 19).

De $V$. serranoi se separa fácilmente porque las antenas en el nuevo taxon alcanzan el ápice elitral, no así en $V$. serranoi y por la puntuación de la cabe$\mathrm{za}$, pronoto y élitros que determinan que la superfi- 


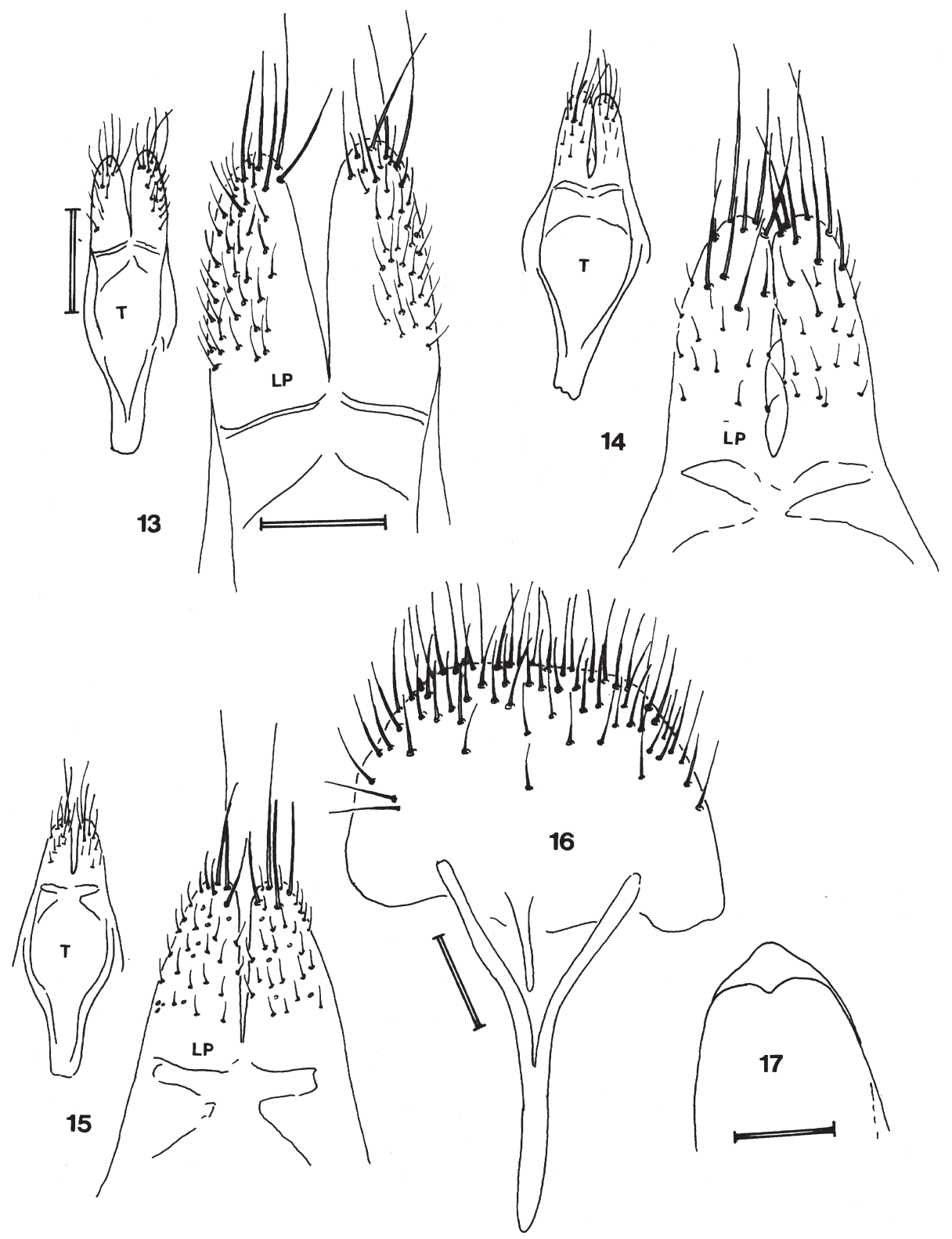

Figs. 13-17.- Vesperus jertensis sp. nov., macho: 13-15) variabilidad del tegmen (T) [Escala= 0,4 mm] y lóbulos parameroides (LP) $[$ Escala $=0,17 \mathrm{~mm}]$; 16) pigidio [Escala $=0,33 \mathrm{~mm}]$; 17) ápice del pene [Escala $=0,14 \mathrm{~mm}$.

Figs. 13-17.- Vesperus jertensis sp. nov., male: 13-15) variability of tegmen (T) [Scale=0,4 mm] and parameroid lobes (LP) $[$ Scale $=0,17 \mathrm{~mm}] ; 16)$ pygidium $[$ Scale $=0,33 \mathrm{~mm}]$; 17) apex of penis $[$ Scale $=0,14 \mathrm{~mm}]$.

cie cefálica, pronotal y elitral sean brillantes, frente a superficies marcadamente mates en $V$. serranoi. Otro aspecto a tener en cuenta es la separación geográfica existente entre la poblaciones de ambos táxones.
De $V$. conicicollis y $V$. baesuriensis se separa atendiendo al desarrollo ocular y, sobre todo, a la distancia interocular que es menor que la separación de las inserciones antenales, mientras que en $V$. 

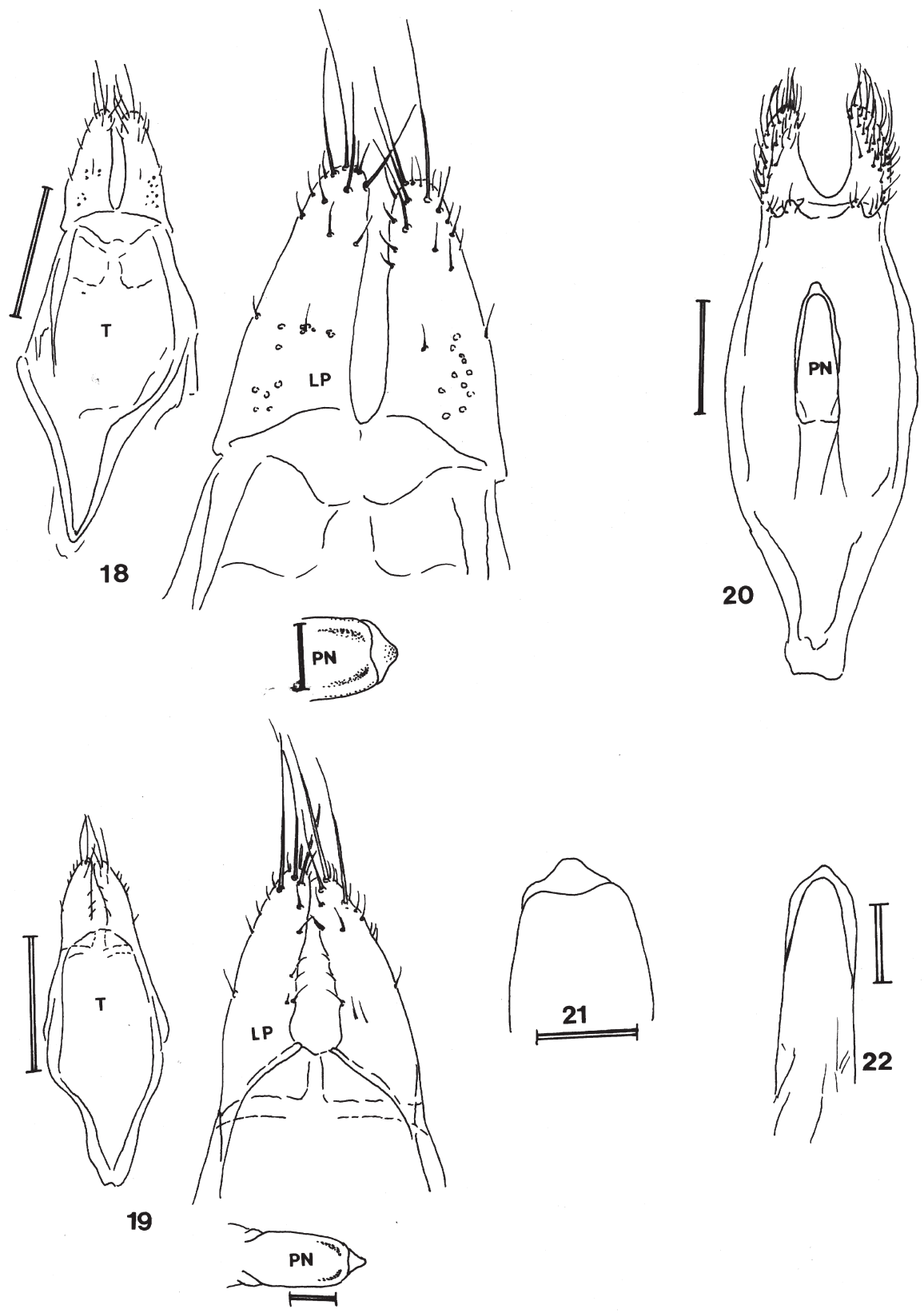

Figs. 18-22.-18) Vesperus baesuriensis Zuzarte, 1985. Paratipo. Tegmen (T) [Escala=0,5 mm], detalle de lóbulos parameroides (LP) y ápice del pene (PN) [Escala=0,2 mm]. 19) Vesperus serranoi Zuzarte, 1985. Paratipo. Tegmen $(\mathrm{T})$ [Escala= 0,5 mm], detalle de lóbulos parameroides (LP) y ápice del pene (PN) [Escala=0,2 mm]. 20) Vesperus sanzi Reitter, 1895. Tegmen y ápice del pene (PN) [Escala=0,5 mm]. 21) Vesperus conicicollis Fairmaire et Coquerel, 1866. Ápice del pene. [Escala=0,6 mm]. 22) Vesperus brevicollis Graells, 1858. Ápice del pene. [Escala=0,4 mm].

Figs. 18-22.-18) Vesperus baesuriensis Zuzarte, 1985. Paratype. Tegmen (T) [Scale= 0,5 mm], parameroid lobes (LP) and apex of penis (PN) $[\mathrm{Scale}=0,2 \mathrm{~mm}]$. 19) Vesperus serranoi Zuzarte, 1985. Paratype. Tegmen (T) [Scale=0,5 mm], parameroid lobes (LP) and apex of penis (PN) [Scale $=0,2 \mathrm{~mm}$. 20) Vesperus sanzi Reitter, 1895. Tegmen and apex of penis (PN) $[\mathrm{Scale}=0,5 \mathrm{~mm}]$. 21) Vesperus conicicollis Fairmaire et Coquerel, 1866. Apex of penix [Scale= 0,6 mm]. 22) Vesperus brevicollis Graells, 1858. Apex of penix $[$ Scale $=0,4 \mathrm{~mm}$ ]. 
jertensis es mayor. Por otra parte normalmente las antenas de $V$. conicicollis y $V$. baesuriensis son apreciablemente más largas.

Todo parece indicar que $V$. serranoi (Algarve) y $V$. jertensis (norte de Cáceres) pueden haberse diferenciado a partir de un antepasado común con $V$. conicicollis, lo que explicaría la posición periférica de las poblaciones de estos Vesperus con respecto al área de distribución más extensa de $V$. conicicollis.

\section{AGRADECIMIENTOS}

Queremos dejar constancia de nuestro agradecimiento a D. Ángel Blázquez (Cáceres) y D. Jesús de Castro (Cáceres) por la confianza demostrada al enviarnos los primeros ejemplares de este nuevo taxon, a D. Antonio José Zuzarte (MonfortePortugal) por el préstamo de material típico de $V$. serranoi y $V$. baesuriensis, y al Dr. M. A. Alonso Zarazaga (Museo Nacional de Ciencias Naturales-Madrid) por las gestiones realizadas para permitirnos el estudio de material típico de $V$. serranoi y por sus atinados comentarios que contribuyeron a la redacción definitiva del presente artículo.

\section{Referencias}

BenSE, U., 1995. Longhorn Beetles. Illustrated key to the Cerambycidae and Vesperidae of Europe. Margraf ed. Weikershim. 512 pp.

ViLLIERS, A., 1974. Notes sur les Vesperus d'Espagne (Col. Cerambycidae). Ann. Soc. Entomol. Fr., (N.S.), 10(3): 535-552.
VIVES, E., 1998. Notas sobre longicornios ibéricos (VIII). Una nueva especie ibérica del género Vesperus Dejean, 1821 (Coleoptera: Cerambycidae). L'Entomologiste, 54(4): 183-191.

ZUZARTE, A., 1985. Contribuição para o conhecimento dos Cleridae, Buprestidae e Cerambycidae de Portugal (Insecta Coleoptera). Descrição de duas novas espécies de Vesperus Latreille (Col. Cerambycidae). Libro de Actas del II Congreso Ibérico de Entomología. Bol. Soc. port. Entomol. (Suplemento $\mathrm{N}^{\mathrm{o}}$ 1): 95-103.
Recibido, el 29-XII-1998

Aceptado, el 7-V-1999

Publicado, el 15-XII-1999 